\title{
Theory of Protostellar Disk Formation
}

\author{
Zhi-Yun Li ${ }^{1}$, Ruben Krasnopolsky ${ }^{2}$ and Hsien Shang ${ }^{2}$ \\ ${ }^{1}$ Astronomy Department, University of Virginia \\ email: zl4h@virginia.edu \\ ${ }^{2}$ Academia Sinica, Institute of Astronomy and Astrophysics and Theoretical Institute for \\ Advanced Research in Astrophysics, Taiwan
}

\begin{abstract}
How large, 100-AU scale, rotationally supported disks form around protostars remains unsettled, both observationally and theoretically. In this contribution, we discuss the theoretical difficulties with disk formation in the presence of a dynamically significant magnetic field and their possible resolutions. These difficulties are caused by the concentration of magnetic field lines close to the forming star by protostellar collapse, and the strong magnetic braking associated with the concentrated field. Possible resolutions include magnetic field-rotation axis misalignment, non-ideal MHD effects, and turbulence. The field-rotation misalignment has been shown to promote disk formation, especially when the field is relatively weak and the misalignment angle is relatively large. Non-ideal MHD effects can enable the formation of small disks at early times. How such disks grow at later times remains to be fully quantified. Turbulence has been found to enable disk formation in a number of simulations, but the exact reason for its beneficial effect is debated.
\end{abstract}

Keywords. stars: formation, accretion disk, magnetic field, turbulence

How disks form is an important, unsolved, problem in star and planet formation. One may expect disks to form as a simple consequence of angular momentum conservation during the hydrodynamic collapse of dense, rotating cores of molecular clouds. However, in the presence of a dynamically significant magnetic field, disk formation becomes more uncertain. We will first discuss the theoretical difficulties with disk formation in magnetized dense cores in the ideal MHD limit. This is followed by a discussion of possible resolutions of these difficulties, including non-ideal MHD effects, field-rotation misalignment, and turbulence.

\section{Magnetic Braking Catastrophe in Ideal MHD Limit}

The basic difficulty with disk formation in magnetized dense cores can be illustrated most clearly in the strict ideal MHD limit, where the magnetic field lines are perfectly frozen into the core material. In this limit, as a finite amount of mass is accreted onto the central object (the protostar), a finite amount of magnetic flux will be dragged into the object as well. The magnetic flux accumulated at the center forms a magnetic split monopole, with the field lines fanning out radially. As a result, the magnetic field strength increases rapidly with decreasing distance to the center, as $B \propto r^{-2}$. The magnetic energy density, which is proportional to the field strength squared, increases with decreasing radius even more rapidly, as $E_{B} \propto r^{-4}$. This increase is faster than, for example, the energy density of the accretion flow, which can be estimated approximately from spherical free-fall collapse as $E_{\mathrm{ff}} \propto r^{-5 / 2}$. As the infalling material approaches the central object, it will become completely dominated by the magnetic field sooner or later. The strong magnetic field at small radii is able to remove all of the angular momentum 
in the collapsing flow, leading to the so-called "magnetic braking catastrophe" for disk formation (Galli et al. 2006).

The catastrophic braking of disks in magnetized dense cores in the ideal MHD limit was also found in many numerical as well as semi-analytic calculations. Krasnopolsky \& Königl (2002) were the first to show semi-analytically, using the so-called "thin-disk" approximation, that the formation of rotationally supported disks (RSDs hereafter) can be suppressed if the efficiency of magnetic braking is large enough. However, the braking efficiency was parametrized rather than computed self-consistently. Similarly, Dapp \& Basu (2010) and Dapp et al. (2012) demonstrated that, in the absence of any magnetic diffusivity, a magnetic split-monopole is produced at the center and RSD formation is suppressed, again under the thin-disk approximation.

The catastrophic braking that prevents the formation of RSDs during the protostellar accretion phase has been found in several subsequent 2D and 3D ideal MHD simulations (Tomisaka 1998; Allen et al. 2003; Mellon \& Li 2008; Hennebelle \& Fromang 2008; Duffin \& Pudritz 2009; Seifried et al. 2012; Santos-Lima et al. 2012). Mellon \& Li (2008), in particular, formulated the disk formation problem in the same way as Allen et al. (2003), by adopting a self-similar rotating, magnetized, singular isothermal toroid as the initial configuration. Although idealized, the adopted initial configuration has the advantage that the subsequent core collapse should remain self-similar. The self-similarity provides a useful check on the correctness of the numerically obtained solution. They found that the disk formation was suppressed by a field as weak as $\lambda=13.3$, where $\lambda$ is the massto-flux ratio of the dense core in units of the critical value $1 /(2 \pi \sqrt{G})$.

It should be noted that ideal MHD simulations of core collapse and star/disk formation are intrinsically difficult to converge. This is because the collapse tends to produce a split magnetic monopole near the center, which has a sharp field reversal near the equatorial plane that is prone to (often violent) numerical reconnection ( $\mathrm{Li}$ et al. 2014; Masson et al. 2015). When and where the reconnection occurs depends on numerical resolution, which makes it difficult, perhaps not possible, to obtain numerically converged solutions. Nevertheless, both analytic arguments and currently available numerical simulations support the notion that, in the ideal MHD limit, catastrophic braking makes it difficult to form rotationally supported disks in (laminar) dense cores magnetized to a realistic level (with a typical $\lambda$ of a few; Troland \& Crutcher 2008). Additional effects must be included in order to enable disk formation.

\section{Non-ideal MHD Effects}

In the ideal MHD limit, the flux-freezing condition leads to a magnetic flux problem for the central stellar object, which then results in a magnetic braking catastrophe. Non-ideal MHD effects can in principle break the flux-freezing condition, and that gives a chance to solve the magnetic flux problem, and potentially the magnetic braking catastrophe. The three more well-known non-ideal MHD effects are ambipolar diffusion (AD), the Hall effect, and Ohmic dissipation (see Armitage 2011 for a review). Roughly speaking, in the simplest case of an electron-ion-neutral medium, both ions and electrons are well tied to the magnetic field in the ambipolar diffusion regime. In the Hall regime, electrons remain well tied to the field, but not ions. At the highest densities, both electrons and ions are knocked off the field lines by collisions before they finish a complete gyration; in such a case, Ohmic dissipation dominates. This simple picture is complicated by dust grains, whose size distribution in dense cores is relatively uncertain, but which can become the dominant charge carriers. Under typical cloud conditions, ambipolar diffusion dominates over the other two effects at densities typical of cores (e.g., Nakano et al. 2002, Kunz \& 
Mouschovias 2010). It is the most widely studied non-ideal MHD effect in the context of core formation and evolution in the so-called "standard" picture of low-mass star formation out of magnetically supported clouds (Nakano 1984; Shu et al. 1987; Mouschovias \& Ciolek 1999). It is the effect that we will first concentrate on.

Ambipolar diffusion enables the magnetic field lines that are tied to the ions to drift relative to the bulk neutral material. In the context of disk formation, its most important effect is to redistribute the magnetic flux that would have been dragged into the central object in the ideal MHD limit to a circumstellar region where the magnetic field strength is greatly enhanced ( $\mathrm{Li} \&$ McKee 1996) solving the problem of the central magnetic flux, but not necessarily the problem of excessive magnetic braking. Indeed, the enhanced circumstellar magnetic field is strong enough to drive a hydromagnetic shock into the protostellar accretion flow (Li \& McKee 1996; Ciolek \& Königl 1998; Contopoulos et al. 1998; Krasnopolsky \& Königl 2002; Tassis \& Mouschovias 2007; Dapp et al. 2012). Krasnopolsky \& Königl (2002) showed semi-analytically, using the 1D thin-disk approximation, that disk formation may be suppressed in the strongly magnetized post-shock region if the magnetic braking is efficient enough. The braking efficiency, parametrized in Krasnopolsky \& Königl (2002), was computed self-consistently in the 2D (axisymmetric) simulations of Mellon \& Li (2009), which were performed under the usual assumption of ion density proportional to the square root of neutral density. 3D simulations of AD were performed by Duffin \& Pudritz (2009) using a specially developed, single fluid AMR code (Duffin \& Pudritz 2009) as well as by a two fluid SPH code (Hosking \& Whitworth 2004). Mellon \& Li (2009) found that ambipolar diffusion does not weaken the magnetic braking enough to allow rotationally supported disks to form for realistic levels of cloud core magnetization and cosmic ray ionization rate. In many cases, the magnetic braking is even enhanced. These findings were strengthened by Li et al. (2011), who computed the ion density self-consistently using the simplified chemical network of Nakano and collaborators (Nakano et al. 2002; Nishi et al. 1991) that includes dust grains. We should note that RSD formation may still be possible if the cosmic ray ionization rate can be reduced well below the canonical value of $10^{-17} \mathrm{~s}^{-1}$ (Mellon \& Li 2009), through for example the magnetic mirroring effect, which may turn a large fraction of the incoming cosmic rays back before they reach the disk-forming region (Padovani et al. 2013, 2014). Masson et al. (2015) showed that ambipolar diffusion in their simulations lowered the maximum field strength obtained close to the central object compared to the case of ideal MHD, and enabled the formation of rotation-dominated, spiral-like structure of order 50-80 AU in radius for a magnetized, $\lambda \approx 4$, core. Whether this structure can be called a rotationally supported disk or not depends on how the disk is defined, which is somewhat ambiguous. More work is needed to ascertain the role of ambipolar diffusion in disk formation.

As the density increases, the Hall effect tends to become more important. It is less explored than ambipolar diffusion in the star formation literature. A unique feature of this effect is that it can actively increase the angular momentum of a collapsing, magnetized flow through the so-called "Hall spin-up" (Wardle \& Ng 1999). In the simplest case of electron-ion-neutral fluid, the spin-up is caused by the current carriers (the electrons) moving in the azimuthal direction, generating a magnetic torque through field twisting; the toroidal current is produced by gravitational collapse, which drags the poloidal field into a pinched, hourglass-like configuration. The Hall spin-up was studied numerically by Krasnopolsky et al. (2011) and semi-analytically by Braiding \& Wardle (2012a,b). Krasnopolsky et al. (2011) showed that a rotationally supported disk can form even in an initially non-rotating core, provided that the Hall coefficient is large enough. Interestingly, when the direction of the initial magnetic field in the core is flipped, the disk 
rotation is reversed. The Hall effect, although dynamically significant, does not appear capable of forming a large (100-AU scale) rotationally supported disk under typical dense core conditions according to Li et al. (2011), at least at relatively late times where substantial mass has accreted onto the central stellar object. It does appear to facilitate the formation of a rotationally supported structure during the first core formation at early times (Tsukamoto et al. 2015a).

Ohmic dissipation becomes the dominant nonideal MHD effects at high densities (e.g., Nakano et al. 2002). It has been investigated by different groups in connection with disk formation. Shu et al. (2006) studied semi-analytically the effects of a spatially uniform resistivity on the magnetic field structure during the protostellar mass accretion phase. They found that, close to the central object, the magnetic field decouples from the collapsing material and becomes more or less uniform. They suggested that a rotationally supported disk may form in the decoupled region, especially if the resistivity is higher than the classic (microscopic) value. This suggestion was confirmed by Krasnopolsky et al. (2010; see also Santos-Lima et al. 2012), who found numerically that a large, $10^{2} \mathrm{AU}$-scale, Keplerian disk can form around a $0.5 \mathrm{M}_{\odot}$ star, provided that the resistivity is of order $10^{19} \mathrm{~cm}^{2} \mathrm{~s}^{-1}$ or more; such a resistivity is significantly higher than the classic (microscopic) value over most of the density range relevant to disk formation.

Machida \& Matsumoto (2011) and Machida et al. (2011) studied disk formation in magnetized cores including only the classic value of resistivity estimated from Nakano et al.'s (2002) numerical results. The former study found that a relatively small, 10 AUscale, rotationally supported disk formed within a few years after the formation of the stellar core. Inside the disk, the density is high enough for magnetic decoupling to occur due to Ohmic dissipation. This work was extended to much later times by Machida et al. (2011), who included a central sink region in the simulations. They concluded that the small RSD can grow to large, $10^{2}$-AU size at later times, especially after most of the envelope material has fallen onto the disk and the central object. A caveat, pointed out by Tomida et al. (2013; see also Dapp \& Basu 2010), is that they used a form of induction equation that is, strictly speaking, inappropriate for the non-constant resistivity adopted in their models; it may generate magnetic monopoles that are subsequently advected away using the Dedner's method (Dedner et al. 2002). This deficiency was corrected in Tomida et al. (2013), who carried out radiative MHD simulations of magnetized core collapse to a time shortly ( $\sim 1$ year) after the formation of the second (protostellar) core. They found that the formation of a (small, AU-scale) rotationally supported disk was suppressed by magnetic braking in the ideal MHD limit but was enabled by Ohmic dissipation at this early time; the latter result is in qualitative agreement with Machida \& Matsumoto (2011) and Machida et al. (2011), although it remains to be seen how the small disks in Tomida et al. (2013)'s simulations evolve further in time.

Dapp \& Basu (2010) studied the effects of Ohmic dissipation on disk formation semianalytically, using the "thin-disk" approximation for the mass distribution and an approximate treatment of magnetic braking. The approximations enabled them to follow the formation of both the first and second core. They found that a small, sub-AU, rotationally supported disk was able to form soon after the formation of the second core in the presence of Ohmic dissipation; it was suppressed in the ideal MHD limit, in agreement with the later 3D simulations of Tomida et al. (2013). This work was extended by Dapp et al. (2012) to include a set of self-consistently computed charge densities from a simplified chemical network and ambipolar diffusion. They showed that their earlier conclusion that a small, sub-AU scale, RSD is formed through Ohmic dissipation holds even in the presence of a realistic level of ambipolar diffusion. This conclusion appears reasonably secure in view of the broad agreement between the semi-analytic work and 
numerical simulations (see also Tomida et al. 2015 and Tsukamoto et al. 2015b). When and how such disks grow to the much larger, $10^{2} \mathrm{AU}$-scale, size deserve to be explored more fully.

\section{Magnetic Field-Rotation Misalignment}

Misalignment between the magnetic field and rotation axis as a way to form large RSDs has been explored extensively by Hennebelle and collaborators (Hennebelle \& Ciardi 2009; Ciardi \& Hennebelle 2010; Joos et al. 2012; see also Machida et al. 2006, and Boss \& Keiser 2013). The misalignment is expected if the angular momenta of dense cores are generated through turbulent motions (e.g., Burkert \& Bodenheimer 2000; Myers et al. 2013; Joos et al. 2013). Plausible observational evidence for it was recently uncovered by Hull et al. (2013) using CARMA, who found that the distribution of the angle between the magnetic field on the $10^{3} \mathrm{AU}$-scale and the bipolar outflow axis (taken as a proxy for the rotation axis) is consistent with being random. If true, it would imply that in half of the sources the two axes are misaligned by an angle greater than $60^{\circ}$. Joos et al. (2012) found that such a large misalignment enables the formation of RSDs in moderately magnetized dense cores with a dimensionless mass-to-flux ratio $\lambda$ of $\sim 3-5$; RSD formation is suppressed in such cores if the magnetic field and rotation axis are less misaligned. They attributed the disk formation to a reduction in the magnetic braking efficiency induced by large misalignment. In more strongly magnetized cores with $\lambda \lesssim 2$, RSD formation is suppressed independent of the misalignment angle, whereas in very weakly magnetized cores RSDs are formed for all misalignment angles.

Based on the work of Hull et al. (2013) and Joos et al. (2012), Krumholz et al. (2013) estimated that the field-rotation misalignment may enable the formation of large RSDs in $\sim 10-50 \%$ of dense cores. If the upper range is correct, the misalignment would go a long way toward solving the problem of excessive magnetic braking in protostellar disk formation.

Li et al. (2013) carried out simulations similar to those of Joos et al. (2012), except for the initial conditions. They confirmed the qualitative result of Joos et al. (2012) that the field-rotation misalignment is conducive to disk formation. In particular, large misalignment weakens the strong outflow in the aligned case and is a key reason behind the formation of RSDs in relatively weakly magnetized cores. For more strongly magnetized cores with $\lambda \lesssim 4$, RSD formation is suppressed independent of the degree of misalignment. This threshold value for the mass-to-flux ratio is about a factor of 2 higher than that obtained by Joos et al. (2012). The difference may come, at least in part, from the different initial conditions adopted: uniform density with a uniform magnetic field for $\mathrm{Li}$ et al. (2013) and a centrally condensed density profile with a nonuniform but unidirectional field for Joos et al. (2012); the magnetic braking is expected to be more efficient at a given (high) central density for the former initial configuration, because its field lines would become more pinched, with a longer lever arm for braking. Whether there are other factors that contribute significantly to the above discrepancy remains to be determined.

If the result of Li et al. (2013) is correct, then a dense core must have both a large fieldrotation misalignment and a rather weak magnetic field in order to form a RSD. This dual requirement would make it difficult for the misalignment alone to enable disk formation in the majority of dense cores, which are typically rather strongly magnetized according to Troland \& Crutcher (2008, with a median mass-to-flux ratio of $\lambda \sim 2$ ). In a more recent study, Crutcher et al. (2010) argued, based on Bayesian analysis, that a fraction of dense cores could be very weakly magnetized, with a dimensionless mass-to-flux ratio $\lambda$ well 
above unity (see Bertram et al. 2012 for additional arguments for weak field, including field reversal). However, since the median mass-to-flux ratio remains unchanged for the different distributions of the total field strength assumed in Crutcher's (2012) Bayesian analysis, it is unlikely for the majority of dense cores to have $\lambda$ much greater than the median value of 2 . For example, Li et al. (2013) estimated the fraction of dense cores with $\lambda>4$ at $\sim 25 \%$. There is also concern that the random distribution of the fieldrotation misalignment angle found by Hull et al. (2013) on the $10^{3}$ AU scale may not be representative of the distribution on the larger core scale. Indeed, Chapman et al. (2013) found that the field orientation on the core scale (measured using a single-dish telescope) is within $\sim 30^{\circ}$ of the outflow axis for 3 of the 4 sources in their sample (see also Davidson et al. 2011); the larger angle measured in the remaining source may be due to projection effects because its outflow axis lies close to the line-of-sight. If the result of Chapman et al. (2013) is robust and if the outflow axis reflects the rotation axis, dense cores with large misalignment between the magnetic and rotation axes would be rare. In such a case, it would be even less likely for the misalignment to be the dominant mechanism for disk formation.

\section{Turbulence}

Turbulence is a major ingredient for star formation (see reviews by, e.g., Mac Low \& Klessen 2004 and McKee \& Ostriker 2007). It can generate local angular momentum by shear flows and form highly asymmetric dense cores (see results from Herschel observations, e.g., Men'shchikov et al. 2010 and Molinari et al. 2010). There is increasing evidence that it also promotes RSD formation. Santos-Lima et al. (2012) contrasted the accretion of turbulent and laminar magnetized gas onto a pre-existing central star, and found that a nearly Keplerian disk was formed in the turbulent but not laminar case. The simulations were carried out at a relatively low resolution (with a rather large cell size of 15.6 AU; this was halved, however, in Santos-Lima et al. 2013, who found similar results), and turbulence was driven to an rms Mach number of $\sim 4$, which may be too large for low-mass cores. Nevertheless, the beneficial effect of turbulence on disk formation is clearly demonstrated. They attributed the disk formation to the turbulence-induced outward diffusion of magnetic flux, which reduces the strength of the magnetic field in the inner, disk-forming, part of the accretion flow. The simulations of Seifried et al. (2012b, 2013) also show turbulence allowing disk formation, but these authors propose a different mechanism, in which the turbulence-induced tangling of field lines and strong local shear are mainly responsible for the disk formation: the disorder of the magnetic field weakens its braking efficiency and the shear enhances rotation. However, the connection between the proposed mechanisms and the simulations remains to be fully quantified. It is possible that the results of Seifried et al. (2012b) are compatible with magnetic misalignment (Joos et al. 2013) or magnetic diffusion (Santos-Lima et al. 2013), either due to the mechanism advocated by Santos-Lima et al. (2012) or numerical diffusion, especially during the formation of sink particles.

Similarly, Myers et al. (2013) also observed formation of a nearly Keplerian disk in their radiative MHD simulation of a turbulent massive $\left(300 M_{\odot}\right)$ core, although they refrained from discussing the origin of the disk in detail since it was not the focus of their investigation. Joos et al. (2013) investigated the effects of turbulence of various strengths on disk formation in a core of intermediate mass $\left(5 M_{\odot}\right)$. They found that an initially imposed turbulence has two major effects. It produces an effective diffusivity that enables magnetic flux to diffuse outward, broadly consistent with the picture envisioned in Santos-Lima et al. $(2012,2013)$. It also generates a substantial misalignment between 
the rotation axis and magnetic field direction (an effect also seen in Seifried et al. 2012b and Myers et al. 2013). Both of these effects tend to weaken magnetic braking and make disk formation easier. If the turbulence-induced magnetic diffusion is responsible, at least in part, for the disk formation, then numerical effects would be a concern. In the ideal MHD limit, the diffusion presumably comes from turbulence-enhanced reconnections due to finite grid resolution. Indeed, Joos et al. (2013) reported that their simulations did not appear to be fully converged, with disk masses differing by a factor up to $\sim 2$ in higher resolution simulations. The situation is further complicated by numerical algorithms for treating magnetic field evolution, especially those relying on divergence advection and divergence cleaning, which could introduce additional magnetic diffusion and residual magnetic monopoles. To make further progress, it would be useful to determine when and how the reconnections occur and exactly how they lead to the magnetic diffusion that are apparent in the simulations of Joos et al. (2013), Santos-Lima et al. (2012), Li et al. (2014) and perhaps Seifried et al. (2012b, 2013). An improvement in our understanding of the detailed structure of the turbulence in star forming cores would also help greatly.

In the simulations of Li et al. (2014), disk formation is observed for a turbulent Mach number in the range $0.5-1.0$, the disk being persistent for $\mathrm{M}=1$. A strongly warped pseudodisk was observed in the disk-forming cases, and a mechanism is proposed in which this pseudodisk warping is envisioned as favorable to the formation of a flat Keplerian disk inside. This mechanism can perhaps be also extended to the misalignment scenario, which also shows warped pseudodisks surrounding the Keplerian disks (Li et al. 2013). These simulations have been performed using a constrained transport code, and therefore are essentially free of magnetic monopoles; they are however not free of magnetic diffusion, especially due to the use of a relatively large spherical hole in their simulation grid. In any case, turbulence has been shown to promote disk formation, but this beneficial effect remains to be full understood and quantified.

In summary, there has been substantial progress in identifying the theoretical difficulties in magnetized disk formation and possible resolutions. Much work remains to be done to determine which of these possibilities, if any, is chosen by Nature.

We thank D. Galli, E. de Gouveia Dal Pino, R. Klessen, S. Lizano, R. Pudritz and R. Santos-Lima for useful discussion. This work was supported in part by NASA NNX10AH30G and NNX14AB38G, and NSF AST1313083.

\section{References}

Allen, A., Li, Z.-Y., \& Shu, F. H. 2003, ApJ, 599, 363

Armitage, P. J. 2011, ARAA, 49, 195

Bertram, E., Federrath, C., Banerjee, R., \& Klessen, R. S. 2012, MNRAS, 420, 3163

Boss, A. P. \& Keiser, S. A. 2013, ApJ, 764, 136

Braiding, C. R. \& Wardle, M. 2012, MNRAS, 422, 261

Braiding, C. R. \& Wardle, M. 2012, MNRAS, 427, 3188

Burkert, A. \& Bodenheimer, P. 2000, ApJ, 543, 822

Chapman, N. L., Davidson, J. A., Goldsmith, P. F. et al. 2013, ApJ, 770, 151

Ciardi, A. \& Hennebelle, P. 2010, MNRAS, 409, L39

Ciolek, G. E. \& Königl, A. 1998, ApJ, 504, 257

Contopoulos, I., Ciolek, G. E., \& Königl, A. 1998, ApJ, 504, 247

Crutcher, R. M., Wandelt, B., Heiles, C., Falgarone, E., \& Troland, T. H. 2010, ApJ, 725, 466

Crutcher, R. M. 2012, ARAA, 50, 29

Dapp, W. B. \& Basu, S. 2010, AA, 521, 56

Dapp, W. B., Basu, S., \& Kunz, M. W. 2012, AA, 541, A35

Davidson, J. A., Novak, G., Matthews, T. G. et al. 2011, ApJ, 732, 97 
Dedner, A., et al. 2002, Journal of Computational Physics, 175, 645

Duffin, D. F. \& Pudritz, R. E. 2009, MNRAS, 391, 1659

Duffin, D. F. \& Pudritz, R. E. 2009, ApJL, 706, L46

Galli, D., Lizano, S., Shu, F. H., \& Allen, A. 2006, ApJ, 647, 374

Hennebelle, P. \& Ciardi, A. 2009, AA, 506, L29

Hennebelle, P. \& Fromang, S. 2008, AA, 477, 9

Hosking, J. G. \& Whitworth, A. P. 2004, MNRAS, 347, 1001

Hull, C. L. H., Plambeck, R. L., Bolatto, A. D. et al. 2013, ApJ, 768, 159

Joos, M., Hennebelle, P., \& Ciardi, A. 2012, AA, 543, A128

Joos, M., Hennebelle, P., Ciardi, A., \& Fromang, S. 2013, AA, 554, A17

Krasnopolsky, R. \& Königl, A. 2002, ApJ, 580, 987

Krasnopolsky, R., Li, Z.-Y., \& Shang, H. 2010, ApJ, 716, 1541

Krasnopolsky, R., Li, Z.-Y., \& Shang, H. 2011, ApJ, 733, 54

Krumholz, M. R., Crutcher, R. M., \& Hull, C. L. H. 2013, ApJL, 767, L11

Kunz, M. W. \& Mouschovias, T. C. 2010, MNRAS, 408, 322

Li, Z.-Y., Krasnopolsky, R., \& Shang, H. 2011, ApJ, 738, 180

Li, Z.-Y., Krasnopolsky, R., \& Shang, H. 2013, ApJ, 774, 82

Li, Z.-Y., Krasnopolsky, R., Shang, H., \& Zhao, B. 2014, ApJ, 793, 130

Li, Z.-Y. \& McKee, C. F. 1996, ApJ, 464, 373

Machida, M. N., Inutsuka, S.-i., \& Matsumoto, T. 2006, ApJL, 649, L129

Machida, M. N., Inutsuka, S.-i., \& Matsumoto, T. 2011, PASJ, 63, 555

Machida, M. N. \& Matsumoto, T. 2011, MNRAS, 413, 2767

Mac Low, M.-M. \& Klessen, R. S. 2004, Reviews of Modern Physics, 76, 125

Masson, J., Chabrier, G., Hennebelle, P., Vaytet, N., \& Commerçon, B. 2015, AA, in press.

McKee, C. F. \& Ostriker, E. C. 2007, ARAA, 45, 565

Mellon, R. R. \& Li, Z.-Y. 2008, ApJ, 681, 1356

Mellon, R. R. \& Li, Z.-Y. 2009, ApJ, 698, 922

Men'shchikov, A., et al. 2010, AA, 518, L103

Molinari, S., et al. 2010, AA, 518, L100

Mouschovias, T. C., \& Ciolek, G. E. 1999 in: Magnetic Fields and Star Formation: A Theory Reaching Adulthood, (edited by C. J. Lada and N. D. Kylafis), 305

Myers, A. T., McKee, C. F., Cunningham, A. J., Klein, R. I., \& Krumholz, M. R. 2013, ApJ, 766,97

Nakano, T. 1984, Fundamentals of Cosmic Physics, 9, 139

Nakano, T., Nishi, R., \& Umebayashi, T. 2002, ApJ, 573, 199

Nishi, R., Nakano, T., \& Umebayashi, T. 1991, ApJ, 368, 181

Padovani, M., Hennebelle, P., \& Galli, D. 2013, AA, 560, A114

Padovani, M., Galli, D., Hennebelle, P., Commerçon, B., \& Joos, M. 2014, AA, 571, A33

Santos-Lima, R., de Gouveia Dal Pino, E. M., \& Lazarian, A. 2012, ApJ, 747, 21

Santos-Lima, R., de Gouveia Dal Pino, E. M., \& Lazarian, A. 2013, MNRAS, 429, 3371

Seifried, D., Pudritz, R. E., Banerjee, R. et al. 2012, MNRAS, 422, 347

Seifried, D., Banerjee, R., Pudritz, R. E., \& Klessen, R. S. 2012, MNRAS, 423, L40

Seifried, D., Banerjee, R., Pudritz, R. E. et al. 2013, MNRAS, 432, 3320

Shu, F. H., Adams, F. C., \& Lizano, S. 1987, ARAA, 25, 23

Shu, F. H., Galli, D., Lizano, S., \& Cai, M. 2006, ApJ, 647, 382

Tassis, K. \& Mouschovias, T. C. 2007, ApJ, 660, 388

Tomida, K., Tomisaka, K., Matsumoto, T., Hori, Y., et al. 2013, ApJ, 763, 6

Tomida, K., Okuzumi, S., \& Machida, M. N. 2015, ApJ, 801, 117

Tomisaka, K. 1998, ApJL, 502, L163

Troland, T. H. \& Crutcher, R. M. 2008, ApJ, 680, 457

Tsukamoto, Y., Iwasaki, K., Okuzumi, S., Machida, M. N., \& Inutsuka, S. 2015, ApJ, 810, L26

Tsukamoto, Y., Iwasaki, K., Okuzumi, S., Machida, M. N., \& Inutsuka, S. 2015, MNRAS, 452, 278

Wardle, M. \& Ng, C. 1999, MNRAS, 303, 239 